\title{
Relation between fetal anthropometric parameters and cord blood adiponectin and high-sensitivity C-reactive protein in gestational diabetes mellitus
}

\author{
Mohammad Reza Aramesh', Masoud Dehdashtian', \\ Arash Malekian', Shiva ShahAli ${ }^{2}$, Kobra Shojaei ${ }^{3}$
}

1 Department of Pediatrics, Division of Neonatology, Ahvaz Jundishapur University of Medical Sciences, Ahvaz, IR Iran ${ }^{2}$ Student Research Committee, Ahvaz Jundishapur University of Medical Sciences, Ahvaz, IR Iran ${ }^{3}$ Department of Obstetrics and Gynecology, Ahvaz Jundishapur University of Medical Sciences, Ahvaz, IR Iran

Correspondence to: Shiva ShahAli

Ahvaz Jundishapur University of Medical Sciences, Ahvaz, IR Iran shivashahali@gmail.com

Received on Jan/8/2016

Accepted on Oct/10/2016

\begin{abstract}
Objectives: The objectives were to evaluate the relation between fetal anthropometric parameters and cord blood concentration of adiponectin and high sensitivity C-reactive protein (hs-CRP). Subjects and methods: A total of 104 pregnant women (52 with gestational diabetes mellitus [GDM], 52 with normal glucose tolerance (NGT) participated. Venous cord blood samples were obtained at delivery, centrifuged and the plasma was stored at $-20^{\circ} \mathrm{C}$. The samples were assessed for adiponectin and hs-CRP using the ELISA method. Statistical analysis was done using SPSS software. Results: The adiponectin concentration was higher in the GDM group than in the NGT group $(11.05 \pm 4.1 \mu \mathrm{g} /$ $\mathrm{mL}$ in GDM vs. $5.34 \pm 2.63 \mu \mathrm{g} / \mathrm{mL}$ in NGT, $\mathrm{p}<0.001)$. GDM was also higher in neonates delivered at later gestational ages $(p<0.001$, Pearson correlation $=0.59)$. There was a positive correlation between cord blood adiponectin and birth weight in the GDM group ( $p<0.001$, Pearson correlation $=0.619$ ) but not in the NGT group. There was no significant correlation between adiponectin and infant length or head circumference. There was also no significant difference in cord blood hs-CRP concentration between groups. No relation was found between hs-CRP and newborn anthropometric parameters. Conclusion: In the GDM group, adiponectin concentration was considerably higher and had a positive correlation with the ponderal index and birth weight which was not found in the NGT group. Arch Endocrinol Metab. 2017;61(3):228-32.
\end{abstract}

DOI. 10.1590/2359-3997000000235

\section{INTRODUCTION}

G estational diabetes mellitus (GDM) is impaired glucose tolerance that develops or is first diagnosed during pregnancy (1-3). Recent studies have shown a 20 -fold increase in the prevalence of GDM. A further 4 -fold increase in prevalence is anticipated $(3,4)$ after adoption of the new diagnostic criteria suggested by the International Association of Diabetes and Pregnancy Study Groups (IADPSG) $(1,5)$. The presence of GDM means that many infants are exposed to hyperglycemic conditions in utero which can cause neonatal adiposity and metabolic disorders later in life.

The pathogenesis of GDM and its features that result in obesity and metabolic disorders remain unclear. Recent studies have shown that the inflammatory system may play a role in the development and pathogenesis of GDM (1). Insulin resistance and inflammation are major features of maternal metabolic state in women with GDM. Both of these conditions can affect fetal growth (2). The inflammatory environment alters placental gene transcription and fetal metabolic programming. Genes for lipid metabolism and those for inflammatory pathways are upregulated in the placenta of women with GDM (2). This upregulation increases or unbalances production of inflammatory cytokines and energy metabolism regulatory cytokines such as adipokines $(2,6,7)$, which increases adiposity at birth $(8,9)$ and predisposes the newborn to become overweight and develop metabolic diseases such as impaired glucose tolerance, metabolic syndrome, and cardiovascular disease (2,6-8). Previous studies have focused on the pathophysiologic pathways and cytokine levels in GDM and their possible effect on offspring comorbidities later in life $(1,6,7,10)$.

Adiponectin is a cytokine that is released exclusively by adipocytes in adults $(9,11-13)$. Maternal 
adiponectin has a high molecular weight; therefore, it probably does not pass through the placental barrier; its concentration in the umbilical cord is of fetal origin $(14,15)$. The origin of adiponectin in the fetus is not clearly understood (13). In adults, adiponectin concentration is inversely related to adiposity, but its association with adiposity is poorly understood $(9,11,13)$. Some studies indicate a positive correlation between cord plasma adiponectin concentration and adiposity in infants $(8,12,14,16-20)$ and some have found no significant correlation $(9,15)$.

C-reactive protein (CRP) is an acute phase protein in plasma synthesized by the liver in response to pre-inflammatory cytokines. Its concentration increases 12-24 hours after the commencement of an inflammatory process and preserves its level throughout the inflammation. There is a positive correlation between CRP concentration and insulin resistance and elevated levels of CRP have been reported in GDM. A growing body of evidence suggests that CRP is related to development and progression of cardiovascular disease (21).

The present study evaluated the relationship between fetal anthropometric parameters, cord blood adiponectin and hs-CRP concentration at birth and compared mean cord plasma adiponectin and hs-CRP concentrations in the GDM and normal glucose tolerant (NGT) groups.

\section{SUBJECTS AND METHODS}

This analytical, case-controlled study was conducted at Imam Khomeini Hospital (a university-affiliated hospital) in the city of Ahvaz in Iran. Recruitment began in June 2014 and ended in July 2015. The study protocol was approved by the Research Ethics Committee of Ahvaz Jundishapur University of Medical Sciences. Informed consent was obtained from all patients. Prior to participation, all the participants underwent an oral glucose tolerance test ( 3 hour, $100 \mathrm{~g}$ glucose). Those who had two or more values exceeding the thresholds of the GDM diagnostic criteria as suggested by the National Diabetes Data Group (NDDG) were included in the GDM group. Women who had no values meeting a NDDG criteria were included in the NGT group. Those who had only one value exceeding the threshold of the criteria were excluded from the study (22).

Inclusion criteria were a gestational age at delivery of 35 to 41 weeks, singleton pregnancy, first minute Apgar score $>7$ and uncomplicated delivery. Exclusion criteria were substance abuse by mother (including cigarettes, alcohol, etc.), chronic disease and overt diabetes in the mother and the presence of congenital anomalies.

Eventually 104 pregnant women entered the study ( 52 women in the GDM group and 52 in the NGT group). Of the 52 women with GDM, 38 women were given a $40 \%$ carbohydrate diet to control their GDM and 14 women were treated with insulin.

\section{Clinical and demographic data}

The demographic data was gathered by questionnaire. The height and weight of the pregnant women were measured using a calibrated medical scale and recorded. The body mass index (BMI) was calculated as BMI = weight $(\mathrm{kg}) /$ height $\left(\mathrm{m}^{2}\right)$. The length of the newborns was measured using a calibrated length board and their weight with a calibrated scale. To assess fetal growth pattern, the ponderal index (PI) was calculated as $\left(\mathrm{PI}=\frac{\text { Birth weight }(\mathrm{gr})}{\text { Body lenght }(\mathrm{cm})^{3}} \times 100\right)$.

\section{Lab measurement}

Venous cord blood samples were obtained from the 104 full-term healthy infants. After delivery and cord clamping, $5 \mathrm{~mL}$ of cord blood was collected from the umbilical vein in prepared and heparinized tubes at ambient temperature using the aseptic method. The samples were centrifuged and the plasma was kept frozen and stored at $-20^{\circ} \mathrm{C}$ until analysis.

Adiponectin was assayed using an ELISA kit specific to human adiponectin (Biovendor; Laboratorni Medicina; Czech Republic). Serum adiponectin was measured as micrograms per milliliter $(\mu \mathrm{g} / \mathrm{mL})$. Quantitative high sensitivity C-reactive protein (hsCRP) was assayed using an i-CHROMA kit for fluorescence immunoassay specifically for determination of human hs-CRP (Boditech Med Europe; United Kingdom). Serum hs-CRP was measured as milligram per milliliter $(\mathrm{mg} / \mathrm{mL})$.

\section{Statistics}

The data were analyzed by SPSS 13 software (SPSS; USA). The unpaired student T-test was utilized to assess differences between groups. Simple linear regression was employed to eliminate the influence of other predictor values. Pearson correlation was used to analyze the bivariate correlation between adiponectin levels and anthropometric parameters. 


\section{RESULTS}

All infants were healthy and were born at Imam Khomeini Hospital. The demographic and clinical data of the study population are shown in Table 1. Although there was no statistically significant difference in age, parity and BMI between groups. The offspring of the GDM group had higher adiposity, were more prone to be delivered by cesarean section, and were delivered one week earlier on average than the NGT group (Table 1).

All of the 104 infants were included in the study (51 males and 53 females; gestational age: $35-41$ weeks; birth weight: 2300-4150 g). No significant differences were observed between groups. The mean birth weight and PI were higher in the GDM group than the NGT group (birth weight: $3535.1 \pm 400$ g vs. $3041.7 \pm 350$ g, PI: $2.74 \pm 0.28 \mathrm{~g} / \mathrm{cm}^{3}$ vs. $\left.2.40 \pm 0.18 \mathrm{~g} / \mathrm{cm}^{3} ; \mathrm{p}<0.001\right)$. There was no significant difference between the mean length $(50.50 \pm 1.96 \mathrm{~cm}$ in GDM group vs. $50.14 \pm$ $1.43 \mathrm{~cm}$ in NGT group; $\mathrm{p}=0.29$ ) and head circumference $(34.22 \pm 0.65 \mathrm{~cm}$ in GDM group vs. $34.12 \pm 0.79 \mathrm{~cm}$ in NGT group, $\mathrm{p}=0.5$ ) between groups.
The adiponectin present in the cord blood ranged from 0.8 to $22.30 \mu \mathrm{g} / \mathrm{mL}$. Mean cord blood adiponectin was $11.05 \pm 4.1 \mu \mathrm{g} / \mathrm{mL}$ in the GDM group and $5.34 \pm 2.63 \mu \mathrm{g} / \mathrm{mL}$ in the NGT group and was significantly higher in the GDM group $(\mathrm{p}<0.001)$. In addition, there was a significant correlation between cord blood adiponectin and birth weight in the GDM group $(\mathrm{p}<0.001$, Pearson correlation $=0.619)$. No such relation was found in the NGT group. There was no significant correlation between adiponectin and length or head circumference of the infants.

No difference in adiponectin concentrations was observed according to gender or by mode of delivery between groups. Cord blood adiponectin concentrations were higher at later gestational ages and there was a significant correlation between gestational age and adiponectin concentration $(\mathrm{p}<0.00$; Pearson correlation $=0.59$ ). There was no significant difference in cord blood hs-CRP concentration between groups. No significant relationship was found between hs-CRP and newborn anthropometric parameters.

Table 1. Demographic and clinical data of the study population

\begin{tabular}{|c|c|c|c|c|}
\hline & Whole group & NGT group & GDM group & $P$ value \\
\hline$n$ & 104 & 52 & 52 & \\
\hline \multicolumn{5}{|l|}{ Maternal characteristics: } \\
\hline Age (years) & $27.89 \pm 5.63(17-40)$ & $26.8 \pm 5.05$ & $28.9 \pm 6.02$ & NS \\
\hline BMl & $25.17 \pm 4.89$ & $24.96 \pm 5.24$ & $25.37 \pm 4.53$ & NS \\
\hline \multicolumn{5}{|l|}{ Parity } \\
\hline 0 & 41 & 19 & 22 & \multirow{3}{*}{ NS } \\
\hline 1 & 37 & 21 & 16 & \\
\hline$>1$ & 26 & 12 & 14 & \\
\hline Children (boys/girls) & $51 / 53$ & $27 / 25$ & $24 / 28$ & NS \\
\hline Gestational age at delivery (weeks) & $38.12 \pm 1.46(35.44-41)$ & $38.65 \pm 1.45$ & $37.6 \pm 1.28$ & $<0.001$ \\
\hline $\mathrm{HbA1C}$ & - & - & $5.24 \pm 0.32$ & \\
\hline \multicolumn{5}{|l|}{ Delivery } \\
\hline Vaginal & 93 & 50 & 43 & \multirow[t]{2}{*}{$<0.001$} \\
\hline Caesarian & 11 & 2 & 9 & \\
\hline \multicolumn{5}{|l|}{ Neonatal characteristics: } \\
\hline Birth weight $(\mathrm{kg})$ & $3.28 \pm 0.45(2.30-4.15)$ & $3.04 \pm 0.35$ & $3.53 \pm 0.40$ & $<0.001$ \\
\hline Ponderal index $\left(\mathrm{gr} / \mathrm{cm}^{3}\right)$ & $2.57 \pm 0.29(2.04-3.39)$ & $2.40 \pm 0.18$ & $2.74 \pm 0.28$ & $<0.001$ \\
\hline Birth length (cm) & $50.32 \pm 1.72(47-56)$ & $50.14 \pm 1.43$ & $50.50 \pm 1.96$ & NS \\
\hline Head circumference (cm) & $34.17 \pm 0.72(30-36)$ & $34.12 \pm 0.79$ & $34.22 \pm 0.65$ & NS \\
\hline Cord serum adiponectin $(\mu \mathrm{g} / \mathrm{mL})$ & $8.20 \pm 4.470(0.8-22.30)$ & $5.34 \pm 2.63$ & $11.05 \pm 4.1$ & $<0.001$ \\
\hline Cord serum hsCRP (mg/mL) & $0.19 \pm 0.30(0.1-2.0)$ & $0.19 \pm 0.28$ & $0.19 \pm 0.33$ & NS \\
\hline
\end{tabular}

Data are mean \pm SD; HbA1C: glycosylated hemoglobin; NS: not significant. 


\section{DISCUSSION}

Adiponectin is a cytokine of great importance because it can cause establishment of metabolic diseases. Adiponectin concentration has a reverse correlation with inflammatory diseases such as obesity and cardiovascular disease $(23,24)$. Studies have shown that adiponectin affects insulin sensitivity, $\beta$ oxidation and inflammatory pathways. The fact that adiponectin concentrations increase at later gestational ages suggests a role for it in the early growth and development of the fetus $(9,13)$. The present study revealed that the mean cord blood adiponectin concentration in the GDM group was significantly higher than that in the NGT group. Previous studies have reported lower cord blood adiponectin concentrations in the GDM group (11).

The data showed a significant correlation between PI (Pearson correlation: 0.421), birth weight (Pearson correlation: 0.619 ) and cord blood adiponectin concentration. Increased adiponectin levels may play a role in increased birth weight and PI. Ballesteros and cols. (11) reported similar findings. In contrast, Lindsay and cols. (9) found no significant correlation between adiponectin concentration and anthropometric parameters. Another study suggested a reverse correlation between PI and adiponectin concentration $(11,25)$. Some studies have shown a positive correlation between adiponectin concentration and birth weight $(8,12,14,16-20)$, but few have failed to find a significant correlation $(9,15)$. The current study found a positive correlation between adiponectin concentration and gestational age in the GDM group although there was no such correlation in the NGT group. Ballesteros and cols. (11) reported similar results, but Lindsay and cols. (9) found no significant correlation between gestational age and adiponectin.

There was no significant correlation found for adiponectin concentrations versus delivery mode, which agrees well with the findings of Lindsay and cols. (9). The results also show no difference in adiponectin levels by gender. By contrast, Lindsay and cols. (9) reported a difference by gender for adiponectin concentration. No difference in adiponectin concentration was found between women with GDM who were treated with a $40 \%$ carbohydrate diet and those who were treated with insulin in the present study.

This study found no significant difference in cord blood hs-CRP concentration between groups. These findings were similar to those of Mordwinkin and cols. (26) and Jahromi and cols. (27). No significant relation was found between hs-CRP and newborn anthropometric parameters in the present study. To our knowledge the present study is one of the first to investigate the relation between hs-CRP and newborn anthropometric parameters. No similar studies were found to compare with the results of the present study.

The study had certain limitations. Only Iranian women were involved, so the results cannot be extrapolated to other ethnicities. Moreover, financial limitations prevented adoption of IADSPG criteria for screening and diagnosing GDM. This study is one of few to assess the level of adiponectin and hs-CRP in GDM and their relation with fetal anthropometric parameters. Inflammatory pathophysiology may play a role in the development of GDM (1), but the mechanism is not fully understood. The findings of the previous studies have conflicted. Further investigation is needed to examine whether or not a significant difference exists between cord serum concentration of adiponectin and hs-CRP among women with GDM compared to healthy pregnant controls and investigate their relation to fetal anthropometric parameters. To summarize, a significantly higher adiponectin concentration was found in the GDM group and had a positive correlation with PI and birth weight which was not observed in the NGT group.

Acknowledgments: the authors would like to thank the neonatal resuscitation team for cord blood sampling and the staff of the labor and delivery department of the obstetrics and gynecology ward of Imam Khomeini Hospital, affiliated with Ahvaz Jundishapur University of Medical Sciences, for their help during this research.

Disclosure: this work was financially supported through grant GP93100 from the Vice-Chancellor for Research Affairs of Ahvaz Jundishapur University of Medical Sciences. This study has been extracted from the postgraduate thesis of Dr. Shiva ShahAli and was approved by the Health Research Institute of Ahvaz Jundishapur University of Medical Sciences. The authors declare that there is no conflict of interest.

\section{REFERENCES}

1. Gomes CP, Torloni MR, Gueuvoghlanian-Silva BY, Alexandre SM, Mattar R, Daher S. Cytokine levels in gestational diabetes mellitus: a systematic review of the literature. Am J Reprod Immunol. 2013;69(6):545-57.

2. Mitanchez D, Burguet A, Simeoni U. Infants born to mothers with gestational diabetes mellitus: mild neonatal effects, a long-term threat to global health. J Pediatr. 2013;164(3):445-50.

3. Aramesh MR, Dehdashtian M, Malekian A, Babapour R. comparison of the blood level of leptin in umbilical cord of newborns 
of mothers with gestational diabetes and normal mothers and its relationship with growth indices of newborns. Jentashapir J Health Res. 2015;6(5):e25797.

4. Jenum AK, Mørkrid K, Sletner L, Vange S, Torper JL, Nakstad B, et al. Impact of ethnicity on gestational diabetes identified with the WHO and the modified International Association of Diabetes and Pregnancy Study Groups criteria: a population-based cohort study. Eur J Endocrinol. 2012;166(2):317-24.

5. International Association of Diabetes and Pregnancy Study Groups Consensus Panel, Metzger BE, Gabbe SG, Persson B, Buchanan TA, Catalano PA, Damm P, et al. International association of diabetes and pregnancy study groups recommendations on the diagnosis and classification of hyperglycemia in pregnancy. Diabetes Care. 2010;33(3):676-82.

6. Marseille-Tremblay $C$, Ethier-Chiasson $M$, Forest $J C$, Giguère $Y$, Masse A, Mounier C, et al. Impact of maternal circulating cholesterol and gestational diabetes mellitus on lipid metabolism in human term placenta. Mol Reprod Dev. 2008;75(6):1054-62.

7. Reece EA. The fetal and maternal consequences of gestational diabetes mellitus. J Matern Fetal Neonatal Med. 2010;23(3):199-203.

8. Josefsona JL, Zeissb DM, Rademakerd AW, Metzgere BE. Maternal leptin predicts adiposity of the neonate. Horm Res Paediatr. 2014 81(1):13-9.

9. Lindsay RS, Walker JD, Havel PJ, Hamilton BA, Calder AA, Johnstone FD. adiponectin is present in cord blood but is unrelated to birth weight. Diabetes Care. 2003;26(8):2244-9.

10. American Diabetes Association: Diagnosis and classification of diabetes mellitus. Diabetes Care. 2012;35(Suppl. 1):S64-71.

11. Ballesteros M, Simón I, Vendrell J, Ceperuelo-Mallafré V, Miralles RM, Albaiges $\mathrm{G}$, et al. Maternal and cord blood adiponectin multimeric forms in gestational diabetes mellitus. Diabetes Care. 2011;34:2418-23.

12. Mantzoros CS, Rifas-Shiman SL, Williams CJ, Fargnoli JL, Kelesidis T, Gillman MW. Cord Cord blood leptin and adiponectin as predictors of adiposity in children at 3 years of age: a prospective cohort study. Pediatrics. 2009;123(2):682-9.

13. Courville ABP. A DHA-Functional Food During Pregnancy: Impact on Maternal Dietary Intake, Endocrine Parameters and Markers of Infant Body Composition [Doctoral Dissertation]. Connecticut: University of Connecticut; 2006.

14. ChanTF, Yuan SS, Chen HS, Guu CF, Wu LC, YehYT, et al. Correlations between umbilical and maternal serum adiponectin levels and neonatal birthweights. Acta Obstet Gynecol Scand. 2004(83):165-9.

15. Mantzoros C, Petridou E, Alexe D, Skalkidou A, Dessypris N, Papathoma $E$, et al. Serum adiponectin concentration in relation to maternal and perinatal characteristics in newborns. Eur J Clin Endo. 2004(151):741-6.

16. Brynhildsen J, Sydsjo G, Blomberg M, Claesson I-M, Theodorsson E, Nystrom F, et al. Leptin and adiponectin in cord blood from children of normal weight, overweight and obese mothers. Acta Pædiatrica. 2013;102:620-24.

17. Mazaki-Tovi S, Kanety H, Pariente C, Hemi R, Schiff E, Sivan E. Cord blood adiponectin in large-for-gestational age newborns. Am J Obstet Gynecol. 2005;193 1238-42.

18. Kotani Y, Yokota I, Kitamura S, Matsuda J, Naito E, Kuroda Y. Plasma adiponectin levels in newborns are higher than those in adults and positively correlated with birth weight. Clin Endocrinol. 2004;61:418-23.

19. KamodaT, Saitoh H, Saito M, Sugiura M, Matsui A. Serum adiponectin concentrations in newborn infants in early postnatal life. Pediatr Res. 2004(56):690-3.

20. Pardo IM, Geloneze B, Tambascia MA, Barros-Filho AA. Inverse relationship between cord blood adiponectin concentrations and the number of cigarettes smoked during pregnancy. Diab Obes Met. 2005(7):144-7.

21. Howman RA, Charles AK, Jacques A, Doherty DA, Simmer K, Strunk T, et al. Inflammatory and haematological markers in the maternal, umbilical cord and infant circulation in histological chorioamnionitis. PLoS One. 2012;7(12):1-8.

22. Plagemman A, Harder T, Kohlhoff R, Rohde W, Dorner G. Glucose tolerance and insulin secretion in children of mothers with pregestational IDDM or gestational diabetes. Diabetologia. 1997;18: 1094-100.

23. Ahima R. Metabolic actions of adipocyte hormones: focus on adiponectin. Obesity 2006;14: 9S-15S.

24. Matsuzawa. The metabolic syndrome and adipocytokines. FEBS Letters. 2006;580:2917-21.

25. Tsai PJ, Yu CH, Hsu SP, Lee YH, Chiou CH, Hsu YW, et al. Cord plasma concentrations of adiponectin and leptin in healthy term neonates: positive correlation with birthweight and neonatal adiposity. Clin Endocrinol (Oxf). 2004;61(1):88-93.

26. Mordwinkin NM, Ouzounian JG, Yedigarova L, Montoro MN, Louie SG, Rodgers KE. Alteration of endothelial function markers in women with gestational diabetes and their fetuses. J Matern Fetal Neonatal Med. 2013;26(5):507-12.

27. Jahromi BN, Ahmadi N, Cohan N, Jahromi MRN. Comparison of the umbilical artery blood gas, nucleated red blood cell, Creactive protein, and white blood cell differential counts between neonates of diabetic and nondiabetic mothers. Taiwan J Obstet Gynecol. 2011;50(3):301-5. 